\title{
Double shifted magnetization curves in magnetic bilayers
}

\author{
Ching-Ray Chang ${ }^{\mathrm{a}, *}$, Jyh-Shinn Yang ${ }^{\mathrm{b}}$, J.C.A. Huang ${ }^{\text {c }}$, C.H. Lai ${ }^{\mathrm{d}}$ \\ ${ }^{a}$ Department of Physics, National Taiwan University, Taipei, Taiwan \\ ${ }^{\mathrm{b}}$ Institute of Optoelectronic Sciences, National Taiwan Ocean University, Keelung, Taiwan \\ ${ }^{\mathrm{c}}$ Department of Physics, National ChengKung University, Tainan, Taiwan \\ ${ }^{\mathrm{d}}$ Department of Materials Science \& Engineering, National Tsing Hua University, HsinChu, Taiwan
}

Received 5 January 2001; accepted 11 January 2001

\begin{abstract}
Ultrathin ferromagnetic biaxial films exhibit uniaxial anisotropy induced by underlayer and double shifted magnetization curves. Two possible origins of the double shifted curves are identified from experimental data, namely, the step-induced anisotropy and the exchange-coupled induced interfacial anisotropy. Micromagnetic study of modified astroids with starlike shapes indicates that a large uniaxial anisotropy induced by the interface plays a key role in the formation of double shifted magnetization waves. An additional bias field along the easy axis may enhance the shifted field. The present research status, both experimental and theoretical, is briefly discussed. (c) 2001 Elsevier Science Ltd. All rights reserved.
\end{abstract}

Keywords: A. Magnetic materials; A. Multilayers; D. Magnetic properties

\section{Introduction}

A lot of interesting physics related to the magnetic thin film was discovered within the past decades [1]. In particular, the magnetization of ultrathin films was found to reorient with the variation of the film thickness (for a review see Ref. [2]). Symmetry breaking of the surface atoms produces a significant magnetic surface anisotropy in ultrathin magnetic films $[3,4]$ and the physical properties of ultrathin films are therefore quite different from those the bulk. Moreover, the crystal structure can change in ultrathin films as well. Usually the crystal structure of Co is hexagonal in bulk but it becomes $f c c$ in ultrathin film [5]. In particular, different nonmagnetic substrates will produce different strain and lattice mismatch in magnetic thin film [6,7]. Magnetic multilayered systems attracted great attention since the discovery of giant magnetoresistance in 1988 [8]. Fundamental understanding of magnetism has been recently considerably improved thanks to the fabrication of new artificial magnetic geometry. In this field, in particular, the physics of spin-dependent transport gave rise to the new technology of spintronics $[9,72]$ which usually makes use of the fact that interfacial exchange coupling between the

\footnotetext{
* Corresponding author. Fax: +886-2-2363-9984.

E-mail address: crchang@ @hys.ntu.edu.tw (C.-R. Chang).
}

ferromagnetic and antiferromagnetic layer shifts the hysteresis loops [10,11].

Double shifted magnetization curves were known from micromagnetic calculations already a decade ago $[12,13]$. For a large quartic anisotropy term there are four metastable states of the magnetization along the hard axis instead of only two metastable states as in the case where the quartic term is negligible. The double shifted magnetization curves are very hard to observe in an unbiased situation, since the window of material constants in which the double shifted magnetization curves exist is quite narrow, and can not be adjusted in natural magnetic materials. Moreover, the usual random distribution of easy axes will usually destroy the double shifted magnetization curves, so that this effect is very hard to observe in nanocrystalline films or particulate media.

The study of magnetic bilayers with an ultrathin magnetic film epitaxially grown on another film is another interesting research subject with a wealth of physical phenomena. One of these is the existence of the peculiar double shifted magnetization curves [14-17]. Ultrathin magnetic films behave as a single domain, and their magnetic constants can be tailored by the thickness of the film. Therefore, the materials constants window in which the double shifted magnetization curves are observable can be reached at an appropriate film thickness. Moreover, an external or an 
internal constant bias field applied to the ultrathin film will enlarge the observability window. Even though several different interfacial origins of the double shifted magnetization curves were identified [16,18], the basic micromagnetic understanding of the double-loop formation mechanisms is the same in all cases. A magnetic ultrathin film with biaxial (cubic) anisotropy shows symmetric square magnetization curves along two orthogonal directions [19]. If it is deposited on an another film, or possibly coated with a film, then the induced additional uniaxial anisotropy from the interface may break the symmetry of the magnetization reversal conditions along the two orthogonal directions. The double shifted magnetization curves can be observed [14-17] at an appropriate ratio of the intrinsic cubic anisotropy and of the surface-induced uniaxial anisotropy. An external constant bias enhances the observation possibility of double shifted magnetization curves [20-22], and so does an exchange bias field [23,24].

In this paper we review the current research on modeling, fabrication, and characterization of double shifted magnetization curves in bilayers. We also summarize our recent work on epitaxial NiFe/Mo and $\mathrm{Co} / \mathrm{NiMn}$ bilayers, which display many of the characteristic features of the double shifted magnetization curves. Epitaxial magnetic ultrathin films have a well-defined magnetic anisotropy, and thus a precise comparison between experiments and theory can be made. In our experiments the magnetic hysteresis is examined using the magneto-optical Kerr effect and the Vibrating Sample Magnetometry and is then compared with the Stoner-Wohlfarth model. The micromagnetic model clearly indicates that the interfacial properties of bilayers influence the formation of double shifted magnetization curves. The nucleation conditions of magnetization reversal in bilayers are also studied, and the results indicate that incoherent reversal modes may occur along the bilayer easy axis. In conclusion we briefly discuss magnetic properties beyond the double shifted magnetization curves in bilayers.

\section{Micromagnetic theory}

Both quantum and classical theories of magnetization processes must consider at least three energy terms: exchange, anisotropy and magnetostatic. An even more realistic model should include other energy terms, such as magnetostriction, as well, perhaps as a perturbation. However, even though quantum mechanical theory is the basis of our understanding of magnetism, the resultant complicated Hamiltonians can not solved unless significant approximations are introduced. Therefore a classical micromagnetic theory has been developed in parallel with the quantum mechanical studies of magnetization [25,26].

Micromagnetic theory begins with a 1935 paper of Landau and Lifshitz [27] where the wall structure between two antiparallel domains is discussed, and with the pioneering works of Brown [28-30]. In micromagnetic theory the changes in bulk magnetization due to the action of an applied field are described in terms of the changes in the direction of magnetization at every point, and not by domain growth or domain rotation as is done in the domain theory [31]. The existence of domains and domain walls should result naturally from micromagnetic theory for regions where the spatial variation of magnetization is very slow and very fast, respectively. One of the major aims of the micromagnetic theory is the replacement of the quantum spins by classical magnetization vectors, and, also, the replacement of quantum mechanical exchange interaction with a classical exchange energy term in the limit of continuous materials. The micromagnetic theory can then be used in conjunction with Maxwell's equations, and proved to be very successful in recent numerical micromagnetic studies [32].

\subsection{Coherent rotational model}

The reversal mechanism of single domain region has been treated intensively over the past decades [12,13,33-38]. The celebrated Stoner-Wohlfarth model is usually applied only to the ideal exchange-force dominated situation where it yields the model of coherent rotation. The nucleation field along the easy axis in this case is $H_{\mathrm{n}}=2 K_{1} / M_{\mathrm{s}}$ where $M_{\mathrm{s}}$ denotes the saturation magnetization and $K_{1}$ refers to the first nonvanishing anisotropy constant. However, if the volume of the magnetic material increases, then the coherent rotation requires a very high exchange energy to lock all the spins together. In this case, therefore, other nonuniform reversal modes, such as curling and buckling, appear to reduce the required energy for magnetization reversal $[39,40]$.

However, even within the particle size to which the Stoner-Wohlfarth model is commonly applied there are still discrepancies in the observed and predicted values of the nucleation field $[41,42]$. A possible reason is that higher order anisotropy terms in the Stoner-Wohlfarth model were neglected. Usually these higher order terms can be neglected along the easy axis and at very small deviation angles, but this approximation may not be correct along the hard axis where the deviation angle is large. A rigorous calculation with consideration of the higher anisotropy terms is possible within the coherent rotational model $[12,13,37,38]$.

In the coherent rotational model the magnetic free energy per unit volume is composed of the crystalline anisotropy energy and the Zeeman energy, and is generally given by

$E_{\mathrm{T}}=E_{\mathrm{H}}+E_{\mathrm{C}}=-\vec{H} \cdot \vec{M}+K_{1} \sin ^{2} \theta+K_{2} \sin ^{4} \theta$

These three energy terms, which depend on the angle $\theta$ between the magnetization vector and the axis of crystalline symmetry $\mathbf{u}$ (Fig.1), are described as follows:

\subsubsection{Zeeman energy}

$E_{\mathrm{H}}=-M_{\mathrm{s}} H \cos (\beta-\theta)$, 


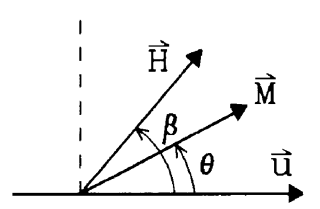

Fig. 1. Schematic geometry used in the micromagnetic model. $\beta$ and $\theta$ are the angles of the applied field and the magnetization making with the axis of crystalline symmetry, respectively.

where $H$ is the external applied field, $M_{\mathrm{s}}$ the saturation magnetization, and $\beta$ is the angle of $H$ with respect to $\mathbf{u}$.

\subsubsection{Crystalline anisotropy energy}

For thin ferromagnetic film, the in-plane uniaxial anisotropy energy may be written as

$E_{\mathrm{C}}=K_{\mathrm{u} 1} \sin ^{2} \theta+K_{\mathrm{u} 2} \sin ^{4} \theta$,

while the in-plane cubic anisotropy is

$E_{\mathrm{C}}=K_{\mathrm{c}} \sin ^{2} \theta \cos ^{2} \theta$,

where $K_{\mathrm{u} 1}$ and $K_{\mathrm{u} 2}$ are the first and second order uniaxial anisotropy constants, and $K_{c}$ is the biaxial anisotropy constant. Obviously, the crystalline anisotropy energy due to contributions of both the uniaxial and the cubic anisotropy is simply $K_{1} \sin ^{2} \theta+K_{2} \sin ^{4} \theta$, where $K_{1}$ and $K_{2}$ may be positive or negative.

To analyze the reversal behavior of magnetic systems it is advantageous to construct a phase diagram, and to determine the existence boundaries of stable states at different fields. By utilizing the normalized quantities

$\epsilon=\frac{E}{2\left|K_{1}\right|}, h_{x}=\frac{H \cos \beta}{H_{\mathrm{k}}}$ and $h_{y}=\frac{H \sin \beta}{H_{\mathrm{k}}}$

with

$H_{\mathrm{k}}=\frac{2\left|K_{1}\right|}{M_{\mathrm{s}}}$,

and

$\alpha=\frac{2 K_{2}}{\left|K_{1}\right|}$,

Eq. (1) is conveniently rewritten in the reduced form

$\epsilon=-h_{x} \cos \theta-h_{y} \sin \theta \pm \frac{1}{2} \sin ^{2} \theta+\frac{1}{4} \alpha \sin ^{4} \theta$,

where + and - refer to $K_{1}>0$ or $K_{1}<0$, respectively. The equilibrium direction of $M$ is determined by the conditions

$$
\begin{aligned}
& \partial \epsilon / \partial \theta=0 \text { and } \partial^{2} \epsilon / \partial \theta^{2}>0, \text { where } \\
& \begin{aligned}
\frac{\partial \epsilon}{\partial \theta}= & h_{x} \sin \theta-h_{y} \cos \theta \pm \sin \theta \cos \theta+\alpha \sin ^{3} \cos \theta \\
\frac{\partial^{2} \epsilon}{\partial \theta^{2}}= & h_{x} \cos \theta+h_{y} \sin \theta \pm\left(\cos ^{2} \theta-\sin ^{2} \theta\right)+\alpha\left(3 \sin ^{2} \cos ^{2} \theta\right. \\
& \left.-\sin ^{4} \theta\right)
\end{aligned}
\end{aligned}
$$

At the critical point, therefore, the locus of critical points in the $h_{x}-h_{y}$ plane is obtained from the equations $\partial \epsilon / \partial \theta=0$ and $\partial^{2} \epsilon / \partial \theta^{2}=0$. In parametric form the loci are given by the equations

$h_{x}=A \cos ^{3} \theta+B \cos ^{5} \theta$,

and

$h_{y}=C \sin ^{3} \theta+B \sin ^{5} \theta$,

where

$A=\mp 1-3 \alpha, B=3 \alpha$ and $C= \pm 1-2 \alpha$.

Eqs. (9a), (9b) and (10) determine the locus of critical field for any values of $K_{1}$ and $K_{2}$.

In the presence of the quartic term the customary StonerWohlfarth astroid becomes modified, and may then be classified into 12 distinct groups, depending the sign and module of $K_{1}$ and $K_{2}$ (Table 1). The equilibrium direction of magnetization can in this case be found by constructing a tangent, closest to the easy axis, to the modified astroid (Fig. 2). At small $\alpha$ the astroid looks like the customary one, except for the cusp contraction or expansion along the $h_{y}$ axis (referred as generalized S-W astroid, Fig. 2I-IV). As the magnitude of $\alpha$ is increased, the cusps turn into swallowtails along the $h_{x}$ or $h_{y}$ axis; this effect represents a spontaneous orientation transition of magnetization (Fig. 2V-VIII). As the magnitude of $\alpha$ increases even further, the astroid become starlike, and the symmetric octapoles are observed only for particular ratios of $K_{2} / K_{1}=-1$ (Fig. 2IX-XII).

\subsection{Nucleation field}

The nucleation field $H_{\mathrm{n}}$ is defined as the external magnetic field at which there is an irreversible switching of magnetization. It is constructive to study the nucleation field for two principal directions with field parallel or perpendicular to the axis of symmetry, i.e. for $\beta=0$ and $\pi / 2$. First, the nucleation field for $\beta=0$ can be obtained by setting $h_{y}=0$ in Eq. (9b), whose solutions then are $\theta=0(\pi)$ or $\sin ^{2} \theta=-C / B$. For simplicity, the decreasing field branch of the loop is only considered in the following. The nucleation field corresponding to $\theta=0$ is $h_{\text {na }}=\mp 1$, where the signs $-(+)$ refer to $K_{1}>0\left(K_{1}<0\right)$. Since $0 \leq \sin ^{2} \theta \leq 1$, the solution of $\sin ^{2} \theta=-C / B$ only exits for case (i) $\alpha>1 / 2$, or $\alpha<-1$ if $K_{1}>0$, and (ii), $\alpha>1$, or $\alpha<-1 / 2$, if 
Table 1

The range of $K_{1}$ and $K_{2}$ defining the 12 distinct regions for modified astroids and nucleation fields in the uniaxial direction $\mathbf{u}\left(H_{n 1}\right.$ and $H_{n 2}$ are the first and second nucleation fields. $\alpha=2 K_{2} \wedge K_{1}$ land $H_{\mathrm{k}}=2\left|K_{1}\right| / M_{\mathrm{s}}$ )

\begin{tabular}{llcl}
\hline Region & Range & $H_{\mathrm{n} 1} / H_{\mathrm{k}}$ & $H_{\mathrm{n} 2} / H_{\mathrm{k}}$ \\
\hline I & $K_{1}>0,0 \leq \alpha \leq 1 / 2$ & -1 & \\
II & $K_{1}<0,-1 / 2 \leq \alpha \leq 0$ & 1 & -1 \\
III & $K_{1}>0,-1 / 3 \leq \alpha \leq 0$ & -1 & -1 \\
IV & $K_{1}<0,0 \leq \alpha \leq 1 / 3$ & 1 & $-2 \alpha[(\alpha+1) / 3 \alpha]^{3 / 2}$ \\
V & $K_{1}>0, \alpha \geq 1 / 2$ & -1 & $2 \alpha[(\alpha-1) / 3 \alpha]^{3 / 2}$ \\
VI & $K_{1}<0, \alpha \leq-1 / 2$ & 1 & -1 \\
VII & $K_{1}>0,-1 \leq \alpha \leq-1 / 3$ & -1 & $2 \alpha[(\alpha+1) / 3 \alpha]^{3 / 2}$ \\
VIII & $K_{1}<0,1 / 3 \leq \alpha \leq 1$ & 1 & $-2 \alpha[(\alpha-1) / 3 \alpha]^{3 / 2}$ \\
IX & $K_{1}>0,-4 \leq \alpha \leq-1$ & -1 & $2 \alpha[(\alpha+1) / 3 \alpha]^{3 / 2}$ \\
X & $K_{1}<0,1 \leq \alpha \leq 4$ & 1 & $-2 \alpha[(\alpha-1) / 3 \alpha]^{3 / 2}$ \\
XI & $K_{1}>0, \alpha \leq-4$ & -1 & \\
XII & $K_{1}<0, \alpha \geq 4$ & 1 & \\
\hline
\end{tabular}
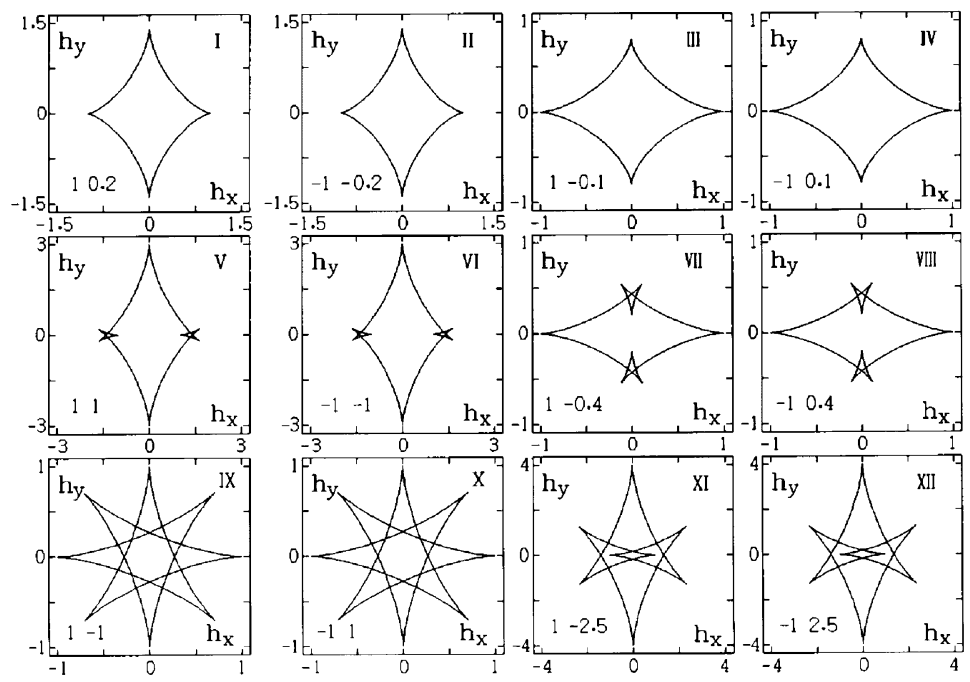

Fig. 2. The modified astroids in 12 distinct regions, where $h_{x}=H_{x} / H_{\mathrm{k}}$ and $h_{y}=H_{y} / H_{\mathrm{k}}$ with $H_{\mathrm{k}}=2$ I

$K_{1} l / M_{\mathrm{s}}$. The values of $K_{1}$ and $K_{2}$ used are shown in the left-bottom of each panel.

$K_{1}<0$. The nucleation field for the case (i) is

$h_{\mathrm{nb}}=-2|\alpha|\left(\frac{\alpha+1}{3 \alpha}\right)^{3 / 2}$,

while in the case (ii),

$h_{\mathrm{nb}}=-2|\alpha|\left(\frac{\alpha-1}{3 \alpha}\right)^{3 / 2}$,

Comparing the magnitudes of $h_{\mathrm{na}}$ and $h_{\mathrm{nb}}$, we find that no hysteresis is present if $K_{1}<0$ and $-1 / 2<\alpha<1$ (Fig. 2II, IV, VIII). On the other hand, there are square-shaped hysteresis loops for $K_{1}>0$ and $-4<\alpha<1 / 2$ (Fig. 2I, III, VII, IX). It is interesting to note that the double shifted magnetization curves appear for $K_{1}<0$ and $\alpha<-1 / 2$ (Fig. 3VI), where the swallowtails occur along the hard direction $\left(h_{x}\right.$ axis).
If the sweeping field is oriented perpendicular to the $\mathbf{u}$ axis, i.e., if $\beta=\pi / 2$, then the magnetic free energy can be rewritten by changing the angular variable as $\phi=\pi / 2-\theta$ :

$E=-\left(K_{1}+2 K_{2}\right) \sin ^{2} \phi+K_{2} \sin ^{4} \phi-H M_{\mathrm{s}} \cos \phi$.

This expression has the same form as that for $\beta=0$, except that $K_{1}$ is replaced by $-\left(K_{1}+2 K_{2}\right)$. Therefore, we can determine the nucleation fields and hysteresis loops for $\beta=\pi / 2$ by using the results derived for $\beta=0$. It is should be noted that the double shifted magnetization curves are also observed for $K_{1}>0$ and $-1<\alpha<-1 / 3$ (Fig. 3VII).

If the field is applied in an arbitrary direction then the nucleation field can be found by simultaneous solution of Eqs. (9a) and (9b). The angular dependence of the nucleation field is in this case given by [13]

$h_{\mathrm{n}}(\beta)=h_{x}(\theta) \cos \beta+h_{y}(\theta) \sin \beta$, 


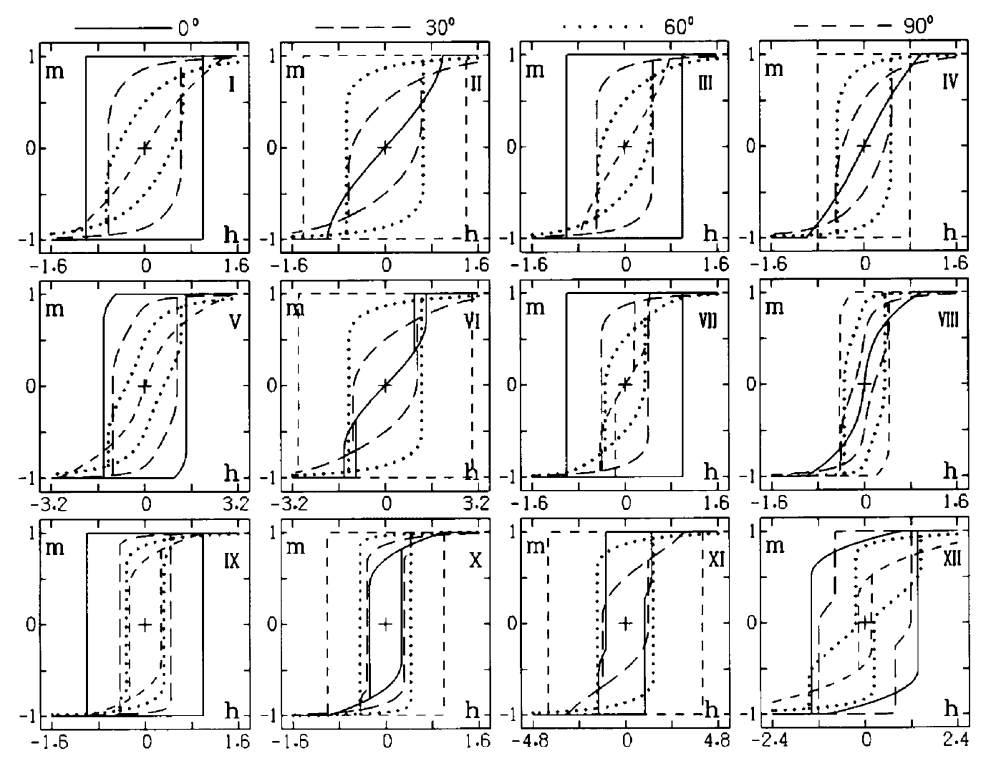

Fig. 3. Calculated hysteresis loops for the 12 regions in Fig. 2, at four different orientations of applied magnetic field, with $H_{\mathrm{k}}=2\left|K_{1}\right| / M_{\mathrm{s}}$. We note that in the regions IX and X, the long dash, dotted, and short dash lines refer to $\beta=15^{\circ}, 30^{\circ}$ and $45^{\circ}$, respectively, otherwise the value of $\beta$ for each line is showed above the panel.

and

$\tan \beta=-\tan ^{3} \theta \frac{C+B \sin ^{2} \theta}{A+B \cos ^{2} \theta}$.

Obviously, for $K_{2}=0$ Eqs. (13a) and (13b) are just the Stoner-Wohlfarth result

$h_{\mathrm{n}}(\beta)=-\frac{1}{\left[\cos ^{2 / 3} \beta+\sin ^{2 / 3} \beta\right]^{3 / 2}}$.

\subsection{Astroid with a bias field}

The double shifted magnetization curves were predicted from micromagnetic calculations. However, as mentioned above, the double shifted loops are very difficult to observe in real magnetic systems, since the window of materials constants in which they occur is quite narrow, and can not be adjusted in natural magnetic materials. Recently, however, the double shifted magnetization curves have been observed along one of the biaxial axis in ultrathin magnetic films [14-17], and by application of a constant external field in the transverse direction [20-22]. These loops can also be observed in the exchange-bias epitaxial $\mathrm{Fe} / \mathrm{MnPd}$ bilayer [24]. Thus, these experimental findings raise the interesting question of how an external or internal constant bias field can change the magnetization process and lead to the occurrence of double shifted loops.

In our analysis here we assume, without loss of generality, that the bias field $H_{\mathrm{b}}$ is oriented along the crystalline symmetry axis $\mathbf{u}$ and that the reduced magnetic free energy is given by

$\epsilon=-\left(h_{x}+h_{\mathrm{b}}\right) \cos \theta-h_{y} \sin \theta \pm \frac{1}{2} \sin ^{2} \theta+\frac{1}{4} \alpha \sin ^{4} \theta$,

where $h_{\mathrm{b}}=H_{\mathrm{b}} / H_{\mathrm{k}}$. Setting $h_{x}{ }^{\prime}=h_{x}+h_{\mathrm{b}}$, we find that Eq. (15) is the same as Eq. (6) for $H_{\mathrm{b}}=0$; therefore, the same astroids should be obtained, apart from the displacement $h_{\mathrm{b}}$ of the applied field along the $h_{x}{ }^{\prime}$ axis. Let us start with the generalized $\mathrm{S}-\mathrm{W}$ astroid. The magnetization process of biased films behaves like that without the $K_{2}$ term $[43,44]$. Depending on the ratio of $H_{\mathrm{b}} / H_{\mathrm{k}}$ and on the field angle, the behavior of magnetization switching can be described by three distinct mechanisms [44-46], but, no double shifted magnetization curves are observed in this kind of bias field.

As mentioned before, for unbiased systems the double shifted loops appear along the hard direction of astroids with swallowtails. When the bias field is introduced, the window for occurrence of the double shifted loops expands to include also starlike astroids. For the sake of simplicity, we only consider here the regions of $K_{1}>0$ and $\alpha<-1 / 3$, and assume that the applied field sweeps perpendicular to the $\mathbf{u}$ axis and that the bias field points along the positive $\mathbf{u}$ axis (Fig. 4). As the field line is located within the range of the swallowtail, a decrease in the applied field from a positive saturation will cause the magnetization to jump to its equilibrium direction close to the easy axis, as crossing the point $\mathrm{A}$ at the boundary. If the field decreases even further, to exceed the point $\mathrm{B}$, the magnetization jumps to an angle of $\theta$ close to $\pi / 2$. Sweeping the field backwards, the magnetization similarly switches its direction at points $\mathrm{A}^{\prime}$ and $\mathrm{B}^{\prime}$. 


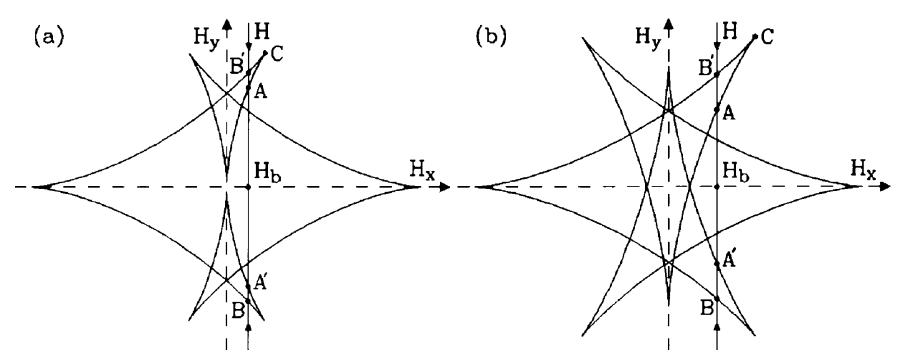

Fig. 4. Use of the astroid demonstrating the position of effective field as the external magnetic field is applied perpendicularly to the uniaxial direction, in combination with the bias field. Here $K_{1}>0$, and $-1<\alpha<-1 / 3$ (a) or $\alpha<-1$ (b).
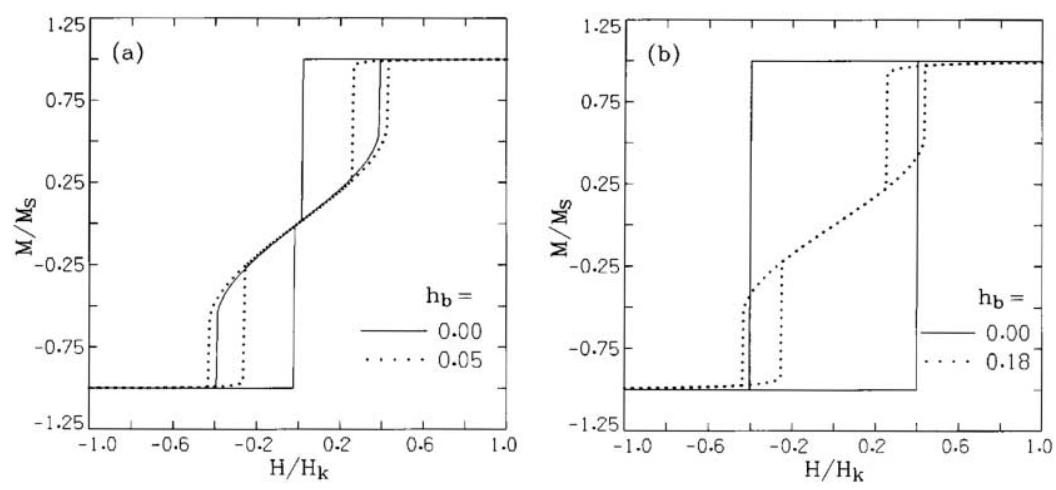

Fig. 5. The hysteresis loops corresponding to the astroids of Fig. 4, with a variation of the bias field. Here $K_{1}>0$, and $\alpha=-0.98$ (a) or $\alpha=-1.50$ (b).

In this manner the double shifted magnetization curve is formed as shown in Fig. 5. To obtain a complete double shifted loop, we further require the points $A\left(A^{\prime}\right)$ to lie above (below) the $h_{x}$ axis and left of point C. From these conditions it follows that the range of the required bias field is

$0 \leq h_{\mathrm{b}}<2|\alpha|\left(\frac{3 \alpha+1}{5 \alpha}\right)^{5 / 2}$ for $-1 \leq \alpha<-1 / 3$,

and

$2|\alpha|\left(\frac{\alpha+1}{3 \alpha}\right)^{3 / 2} \leq h_{\mathrm{b}}<2|\alpha|\left(\frac{3 \alpha+1}{5 \alpha}\right)^{5 / 2}$ for $\alpha<-1$.

It is worthy to mention that the frequently used empirical formula of the shifted field in double shifted loops measurement $[20,21]$ was inconsistent with either the rigorous derivation of the nucleation fields or the numerical results of the shifted fields.

\section{Experiments}

Double-shifted magnetization curves have been observed in various ultrathin magnetic films, e.g. in $\mathrm{Co} / \mathrm{Cu}$ [15], $\mathrm{Co} /$ stepped Cu [14,47-50], Co/NiMn [51], Fe/stepped Ag [16], Fe/stepped W [52], Fe/GaAs [53], Fe/Stepped Cr [54], Fe/
$\mathrm{MnPd}$ [24], NiFe/NiO [23], NiFe/Mo [55], $\mathrm{FeCo} / \mathrm{ZnSe}$ [17] and $\mathrm{FeCo} / \mathrm{GaAs}$ [56] (Table 2). Cubic-anisotropy magnetic thin films grown on either a nonmagnetic or an antiferromagnetic film experience an interfacial induced uniaxial anisotropy. The surface induced bilinear and biquadratic anisotropy together with the intrinsic cubic anisotropy of the magnetic film let the total energy of the magnetic layer follow Eq. (1). The biquadratic anisotropy originates from interfacial coupling which alters the four-fold symmetry to two-fold, and the bilinear anisotropy then provides the internal bias field. Therefore, with variation of the film thickness we can adjust the effective $K_{1}$ and $K_{2}$ in Eq. (1). For example, in the epitaxial $\mathrm{Co} / \mathrm{NiMn}$ system we found that the effective anisotropy constants can be tuned by varying the Co thickness or the annealing time. In this manner the double shifted loops become controllable in the $\mathrm{Co} / \mathrm{NiMn}$ system [51].

The interface induced magnetic anisotropy has attracted wide attention in recent years. Néel [16,57-59] first proposed that atoms in an environment of reduced symmetry, such as those at a surface, will give rise to anisotropy that is different from the bulk anisotropy of the material. In some cases [2-4] the magnetic surface anisotropy is strong enough to overcome the magnetostatic shape energy of the film and results then in a perpendicular magnetic anisotropy. The origins of interfacial anisotropy are very complicated, and can be different in different bilayer system 
$[6,7,18,23,24]$. Two major mechanisms, the step-induced anisotropy, and the exchange-bias induced anisotropy, were proposed to explain the formation of double shifted magnetization curves.

\subsection{Step-induced anisotropy}

In this section, we focus on the interface anisotropy of a magnetic layer grown on a stepped surface. Our recent work on $\mathrm{Ni}_{80} \mathrm{Fe}_{20}$ grown on $\mathrm{Mo}(001)$ stepped surface is also presented here, together with the characteristic double shifted magnetization curves due to the stepped surface effect.

In a magnetic bilayer or multilayer system the interface between a magnetic layer and an underlayer could be (fully or partially) strained. The lattice parameter of a thin film can be different from the bulk values due to the lattice or symmetry mismatch with the underlying template [60$62]$. The continuous-media approximation of the theory of elasticity predicts that psudomorphic growth proceeds with a $1 / t$ relaxation of the thin film parameter, where $t$ is the film thickness $[3,6,7,63]$. This should in turn induce a $1 / t$ dependence of the magnetoelastic contribution to the total magnetic anisotropy of the thin film. Phenomenologically it is thus convenient to write down the interface (or surface) anisotropy term as $2 K_{\mathrm{s}}$, where the factor of 2 account for the top and bottom interfaces of a multilayer system. Most studies use this model to fit experimental data, and separate the contribution of the bulk anisotropy $\left(K_{v}\right)$ and of the interface $\left(2 K_{\mathrm{s}} / t\right)$ term. The effective magnetic anisotropy can thus be expressed as:

$K_{\mathrm{eff}}=K_{\mathrm{v}}-\frac{2 K_{\mathrm{s}}}{t}$,

where $t$ is the magnetic film thickness. The phenomenological model can be applied to the step-induced anisotropy as well. On Co grown on a stepped $\mathrm{Cu}(11 \mathrm{n})$ surface [64], the anisotropy energy density from bulk, surface, and step can be expressed in a form as

$E=K_{\mathrm{v}}-\frac{2 K_{\mathrm{s}}}{t}-\frac{2 K_{\mathrm{sp}}}{t d}$,

where $K_{\mathrm{v}}, K_{\mathrm{s}}, K_{\mathrm{sp}}$ are in units of energy/volume, energy/area, and energy/length, respectively, and $t$ and $d$ are the Co film thickness and terrace width. For Fe grown on a stepped Ag(001) magnetic film, Qiu [16] keeps the functional form of the Néel's model and writes down the energy density of stepped (001) film as

$E=K_{\mathrm{v}}\left(a_{\xi}^{2} a_{\eta}^{2}+a_{\eta}^{2} a_{\zeta}^{2}+a_{\zeta}^{2} a_{\xi}^{2}\right)-\frac{K_{\mathrm{s}}}{t}-\frac{K_{\mathrm{sp}}}{t d}+2 M_{\mathrm{s}} a_{z}$

where $\mathbf{a}$ is the unit vector of magnetization $M$, and $a_{\xi}, a_{\eta}$ and $a_{\zeta}$ are the directional cosines of the [100], [010], and [001] axes as shown in Fig. 6. By varying the magnetic film thickness and the step density the contribution from a single step $\left(K_{\mathrm{sp}}\right)$ was obtained. Characteristic double shifted magnetization curves were observed, with the degree of

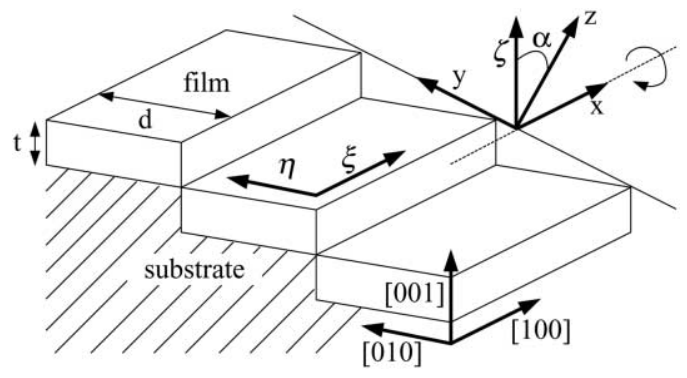

Fig. 6. A Schematic diagram showing the relationship between the crystal frame, film frame, and step geometry of a stepped surface.

the splitting depending on the step density (or vicinal angle). Hayman et. al. [55] employed a single domain switching model to interpret the possible hysteresis loops and magnetization reversal mechanisms. Alternatively, Chang [13] also predicted that for a material with $K_{1}$ satisfying the condition $-6 K_{2}>K_{1}>-2 K_{2}>0$ can exhibit the $M-H$ loop splitting as well.

In our work, the Mo(001) stepped surface was self assembled on sapphire (1-102) substrates. By varying the growth temperature, the $\mathrm{Mo}(001)$ plane can tilt by $3^{\circ}$ from the sapphire (1-102) plane [55]. We note that the self assembly of the $\mathrm{Mo}(001)$ step surface structure is due to the formation of a coherent tilt boundary $[65,66]$ between the Mosapphire interface which, in turn, results from the uniaxial strain exerted by the sapphire substrate along the step (Mo[110]) direction. In addition, the symmetric tilt of the Mo steps was broken by a slight miscut (about $0.5^{\circ}$ ) of the sapphire substrate. The tilt growth and stepped surface of Mo were confirmed by electron and X-ray diffraction.

In order to understand the step interface effect we deposited the $\mathrm{Ni}_{80} \mathrm{Fe}_{20}$ layer at various growth temperatures $\left(T_{\mathrm{g}}\right)$ and used various film thickness on the fresh $\mathrm{Mo}(001)$ stepped surface. The magnetic anisotropy of the $\mathrm{Ni}_{80} \mathrm{Fe}_{20}$ films was then studied by the magneto-optical Kerr effect (MOKE) and a vibrating sample magnetometer (VSM). Note that the probing depth of MOKE is only of about $20 \mathrm{~nm}$ due to the skin depth effect of He-Ne laser.

For the $\mathrm{Ni}_{80} \mathrm{Fe}_{20}$ films grown at low $T_{\mathrm{g}}\left(<200^{\circ} \mathrm{C}\right)$, the easy axis of the $\mathrm{Ni}_{80} \mathrm{Fe}_{20}$ film is directed parallel to the underlying Mo step edge direction $\left(\| \mathrm{Mo}[1-10]\right.$ or $\left.\phi=0^{\circ}\right)$. Along the hard axis $\left(\| \mathrm{Mo}[110]\right.$ or $\left.\phi=90^{\circ}\right)$, the $M-H$ loops reveal a split-loop magnetization rotation process characteristic of magnetic films on stepped surfaces as shown in Fig. 7(a) and (b). The shift field was about 6 Oe for $T_{\mathrm{g}}=100^{\circ} \mathrm{C}$, and decreased to about $3.5 \mathrm{Oe}$ for $T_{\mathrm{g}}=200^{\circ} \mathrm{C}$. At higher $T_{\mathrm{g}}$ $\left(>300^{\circ} \mathrm{C}\right)$, on the other hand, the $\mathrm{Ni}_{80} \mathrm{Fe}_{20}$ films display isotropic like magnetic behavior with much enhanced coercive field as shown for example in Fig. 7(c) and (d). AFM scans reveal that the surface roughness of the $\mathrm{Ni}_{80} \mathrm{Fe}_{20}$ films increases with increasing $T_{\mathrm{g}}$. The growth temperature dependence of the surface roughness together with the coercive field of the $\mathrm{Ni}_{80} \mathrm{Fe}_{20}$ films is shown in Fig. 8. We believe 

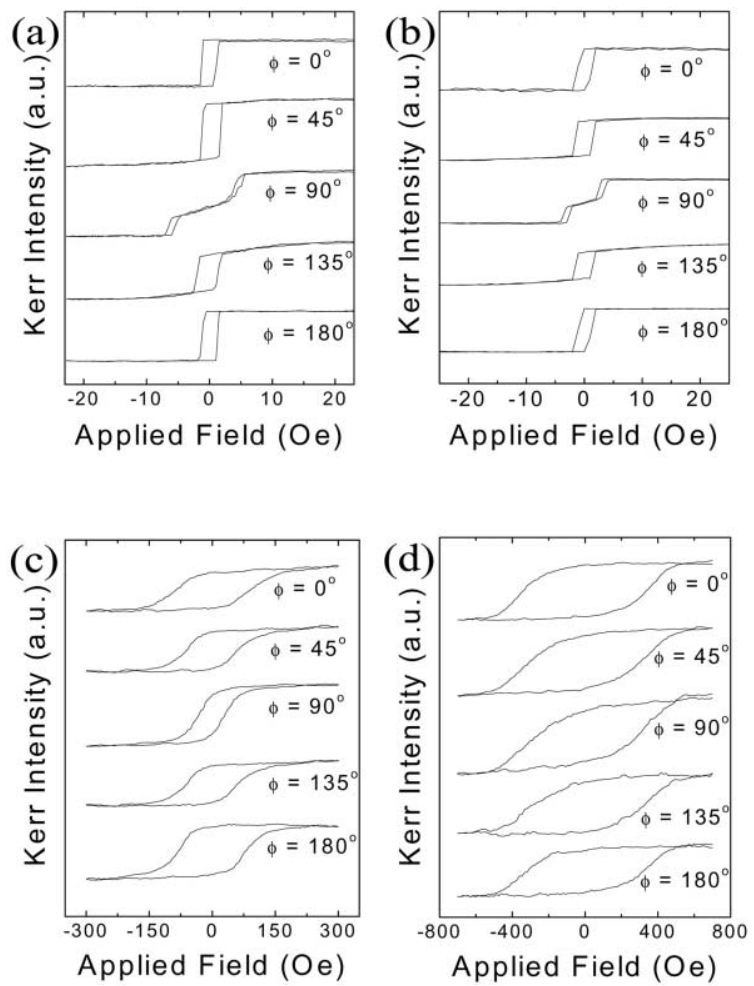

Fig. 7. MOKE hysteresis loops of $10 \mathrm{~nm} \mathrm{Ni}_{80} \mathrm{Fe}_{20}$ grown on $\mathrm{Mo}$ stepped surface with $T_{\mathrm{g}}$ of (a) $100^{\circ} \mathrm{C}$, (b) $200^{\circ} \mathrm{C}$, (c) $300^{\circ} \mathrm{C}$, (d) $400^{\circ} \mathrm{C}$. Here $\phi=90^{\circ}$ is parallel to the Mo[110] azimuth.

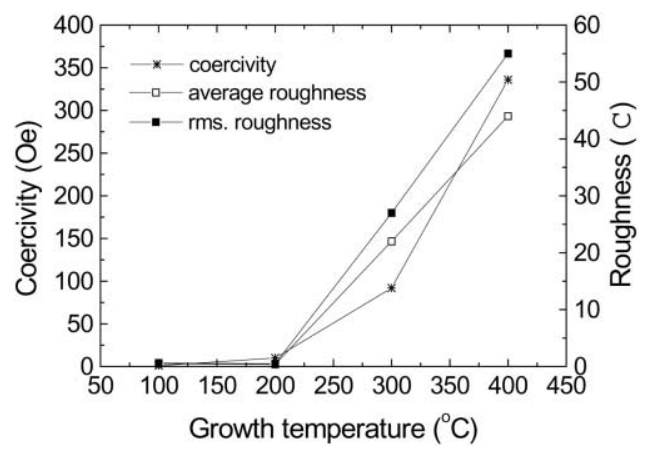

Fig. 8. The surface roughness and coercivity as a function of growth temperature for $\mathrm{Ni}_{80} \mathrm{Fe}_{20}$ films grown on $\mathrm{Mo}(001)$ stepped surface.

that at higher $T_{\mathrm{g}}$ both the surface roughness (due to $3 \mathrm{D}$ nucleation) and the interdiffusion between the $\mathrm{Ni}_{80} \mathrm{Fe}_{20}$ and Mo interface suppress the step-induced magnetic anisotropy, and provide a stronger pinning force for motion of the $\mathrm{Ni}_{80} \mathrm{Fe}_{20}$ domains. Annealing of the low $T_{\mathrm{g}}$ samples was also performed: The loop splitting along the hard axis decreased with increasing annealing temperature, and disappeared completely at about $250^{\circ} \mathrm{C}$. This suggests that the uniaxial anisotropy behavior of the $\mathrm{Ni}_{80} \mathrm{Fe}_{20}$ film is due to
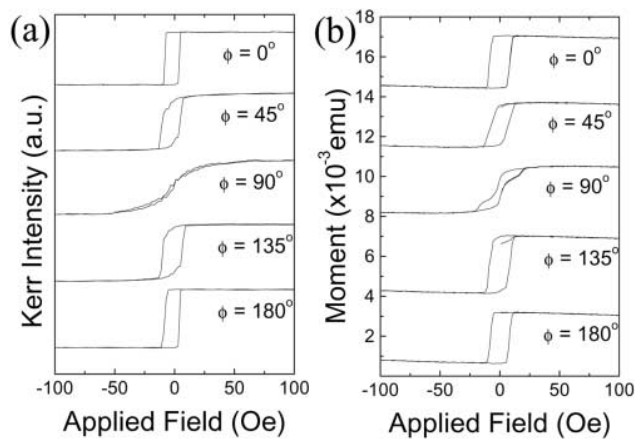

Fig. 9. Hysteresis loops of $50 \mathrm{~nm} \mathrm{Ni}{ }_{80} \mathrm{Fe}_{20}$ film taken by (a) MOKE and (b) VSM technique, respectively.

the step effect which can be smeared out by both high temperature growth and annealing treatment.

In order to separate the interface anisotropy from the bulk anisotropy we study a thick $\mathrm{Ni}_{80} \mathrm{Fe}_{20}$ layer on Mo by using surface sensitive MOKE and bulk sensitive VSM probes. Fig. 9 shows the MOKE and VSM data of a $50 \mathrm{~nm}$ $\mathrm{Ni}_{80} \mathrm{Fe}_{20}$ film grown on Mo stepped surface. From the MOKE measurement one can see that the top $(\sim 20 \mathrm{~nm})$ $\mathrm{Ni}_{80} \mathrm{Fe}_{20}$ layer possesses a uniaxial magnetic anisotropy without loop splitting along hard axis. In contrast, the VSM data (which probe the entire film) show a signal of splitting loops. It is obvious that the split loops originate at the $\mathrm{Ni}_{80} \mathrm{Fe}_{20} / \mathrm{Mo}$ interface. Due to the limited probing depth the split loop cannot be observed by MOKE measurements for thick $(50 \mathrm{~nm}) \mathrm{Ni}_{80} \mathrm{Fe}_{20}$ films.

We conclude that the Mo stepped surface plays an important role in determining the magnetic anisotropy of the subsequently deposited $\mathrm{Ni}_{80} \mathrm{Fe}_{20}$ film. It is worth pointing out that the micro-origin of step-induced magnetic anisotropy may be due to the asymmetry of the in-plane strain of the $\mathrm{Ni}_{80} \mathrm{Fe}_{20}$ film exerted by the (Mo) step edge.

\subsection{Exhange-bias anisotropy}

In the ferromagnetic(F)/antiferromagnetic(AF) bilayers the exchange bias field is usually introduced by an external magnetic field, either by depositing the F layer in a field, or by annealing the bilayers in a field. In both cases the spins of the $\mathrm{F}$ layers tend to align in the direction of the external field and to form a single domain, resulting thus in a unidirectional anisotropy, that is, in a single shifted loop. Exchange anisotropy induced by the residual stress was reported in asdeposited epitaxial (111)NiFe/NiFeMn films grown on (110) $\mathrm{Mo} / \mathrm{Al}_{2} \mathrm{O}_{3}(11-20)$ structural templates by $\mathrm{MBE}$, and the double shifted loops were then observed along the easy axis [67]. In this experiment $\mathrm{NiFe}$ was deposited without the presence of a magnetic field, so that it is no longer a single domain. The in-plane stress arising from the lattice mismatch between $\mathrm{NiFe}$ and Mo introduced a uniaxial 


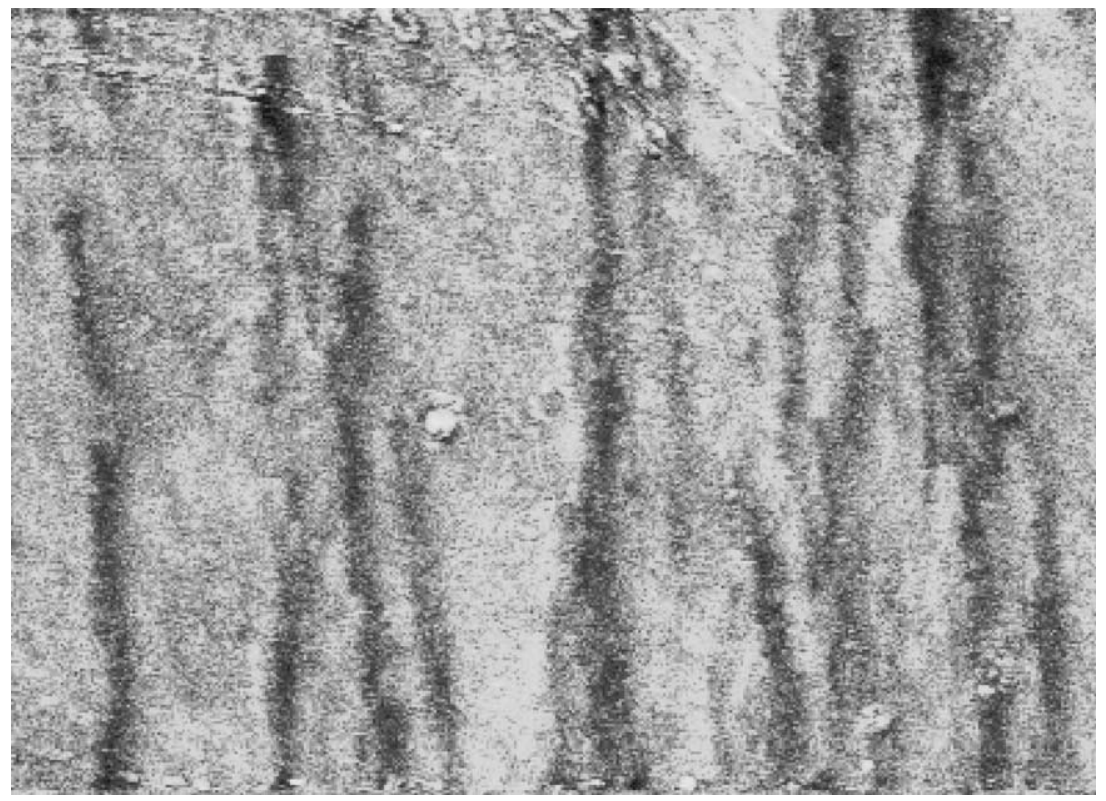

Fig. 10. An MFM image of $\mathrm{Al}_{2} \mathrm{O}_{3} / 20 \mathrm{~nm} \mathrm{Mo} / 8 \mathrm{~nm} \mathrm{NiFe} / 12 \mathrm{~nm} \mathrm{NiFeMn} / 2 \mathrm{~nm}$ Mo. The scale of the image is $40 \times 30 \mathrm{~nm}$. Easy axis is along the short edge of the image.

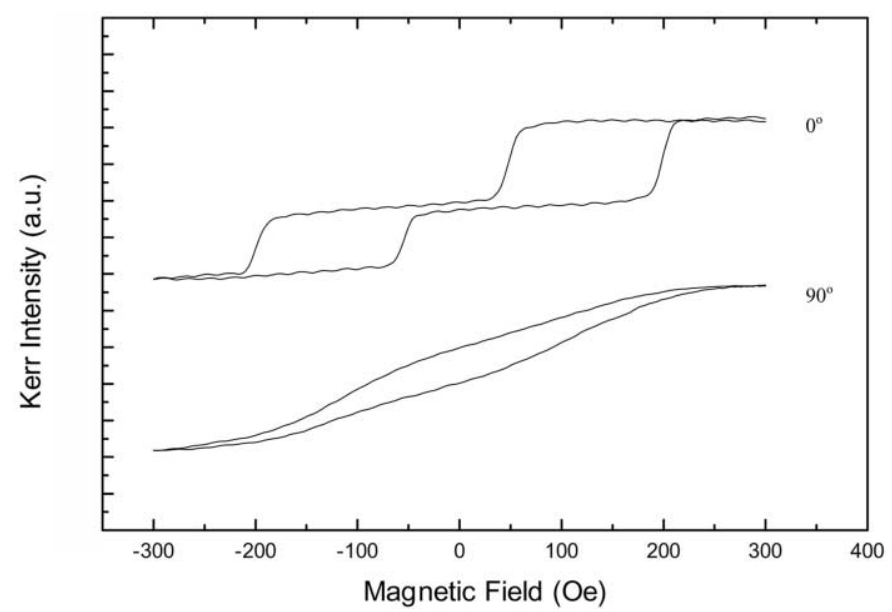

Fig. 11. A double shifted hysteresis loop, measured by MOKE, of the sample $\mathrm{Al}_{2} \mathrm{O}_{3} / \mathrm{Mo}(20 \mathrm{~nm}) / \mathrm{NiFe}(8 \mathrm{~nm}) / \mathrm{NiFeMn}(12 \mathrm{~nm}) / \mathrm{Mo}(2 \mathrm{~nm}) .0^{\circ}$ is along $\mathrm{NiFe}[-211]$, and $90^{\circ}$ is along $\mathrm{NiFe}[01-1]$.

anisotropy in NiFe. Consequently, the spins in NiFe mainly align in this axis (either in positive or negative direction). The MFM image (Fig.10) of the central part of the sample shows stripe-like domains in which the NiFe magnetization is alternately oppositely aligned along the easy axis. Because of the epitaxial growth between $\mathrm{NiFe}$ and $\mathrm{NiFeMn}$ the spins of $\mathrm{NiFeMn}$ at the interface strongly couple to those of $\mathrm{NiFe}$ through interfacial exchange coupling; NiFeMn may therefore break into multi-domains to reduce the interfacial energy between $\mathrm{NiFe}$ and $\mathrm{NiFeMn}$ so that two distinct regions exist in $\mathrm{NiFe}$ with oppositely pinned magnetiza- tions. These give rise to the double shifted loops along the easy axis as shown in Fig. 11.

Double shifted magnetization curves along the hard axis have been observed in epitaxial $\mathrm{NiO} / \mathrm{NiFe}[23]$ and $\mathrm{Fe} /$ MnPd [24] exchange bias systems. Qualitative analysis based on the Stoner-Wohlfarth model provides a preliminary understanding of magnetization reversal in complex anisotropies associated with exchange bias. Detailed quantitative micromagnetic fits of the angular dependence of experimental magnetization curves have been reported in the (100)NiMn/Co system. These measurements clearly 


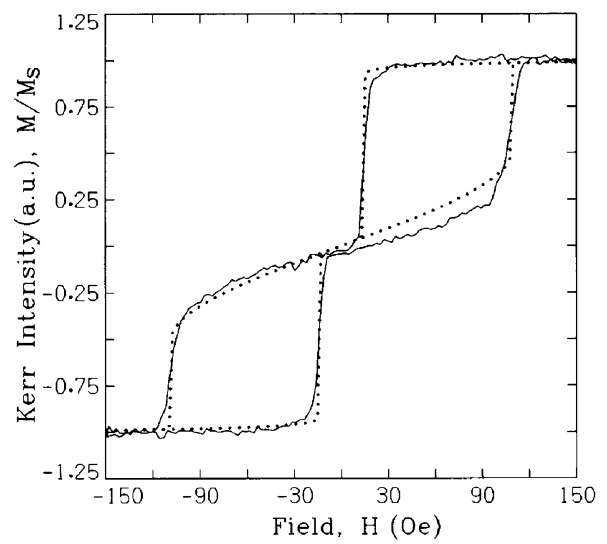

Fig. 12. A micromagnetic fitting of hysteresis loops for NiMn/Co system. The solid line represents the experimental data, and the dotted line is the best-fit curve with $K_{1}=1.92 \times 10^{5} \mathrm{erg} / \mathrm{cm}^{3}$, $K_{2}=-1.58 \times 10^{5} \mathrm{erg} / \mathrm{cm}^{3}$, and $M_{\mathrm{s}}=1400 \mathrm{emu} / \mathrm{cm}^{3}$.

illustrate the origin of the induced anisotropies and explain the double shifted loops [51].

Samples with the structure $\mathrm{Si}(100) / \mathrm{Cu}(15 \mathrm{~nm}) /$ $\mathrm{Ni}_{80} \mathrm{Fe}_{20}(35 \mathrm{~nm}) / \mathrm{NiMn}(50 \mathrm{~nm}) / \mathrm{Co}(\mathrm{t} \mathrm{nm}) / \mathrm{Pd}(15 \mathrm{~nm})$ were grown in an e-beam evaporation system, and the thickness of Co was varied from 5 to $25 \mathrm{~nm}$. Cu was used to initiate (001) epitaxial growth, and thick $\mathrm{Ni}_{80} \mathrm{Fe}_{20}$ was used to prevent the $\mathrm{Cu}$ from diffusing into NiMn [68]. No external field was applied during the deposition, but after the deposition the samples were field-annealed ex-situ in a field of $1000 \mathrm{Oe}$ at $280^{\circ} \mathrm{C}$ in vacuum. This induces the exchange bias field. The as-deposited NiMn is paramagnetic, and Co exhibits a perfect biaxial anisotropy because of the four-fold symmetry. However, after the field annealing along one of the Co easy axis at $280^{\circ} \mathrm{C}$ shifted loops have been observed along the direction of annealing field, indicating the presence of unidirectional anisotropy. The double shifted magnetization curves were observed while measuring loops along the axis perpendicular to the direction of the exchange field. The double shifted loop was attributable to symmetry breaking in the biaxial fcc (001)Co films due to induced interfacial uniaxial anisotropy [51]. The shift field of double loops is larger than the exchange field, suggesting that an extra uniaxial anisotropy can be induced along the field-annealing direction. The exchange coupling between ferromagnetic and antiferromagnetic films induces not only unidirectional, but also uniaxial anisotropy.

The double shifted magnetization curves can be tuned by variation of the Co film thickness or of the field-annealed time. The shifted double magnetization curves only appear in a certain range of Co thickness (between 10 and $15 \mathrm{~nm}$ ) in which the shift field decreases as the thickness increases and is proportional to the exchange bias field. Outside this range of Co thickness the double shifted loops disappear, but the exchange bias remains. This result can be understood within the micromagnetic theory since only appropriate values of the ratio of $K_{2}$ to $K_{1}$ yield the double shifted loops [13]. The induced biquadratic coupling (uniaxial anisotropy) is interficial and strongly depends on the Co thickness. Consequently, only a specific range of the Co thickness values can produce the anisotropy ratio required for the double shifted magnetization curves. Well-fitted micromagnetic calculations of the experimental data, shown in Fig. 12, confirm that biquadratic coupling exists within the NiMn/Co system. The anisotropy constants can then be quantitatively obtained from fitting of the magnetization curves .

Double shifted magnetization curves were also observed in systems with random steps at interface and in miscut substrates where the fourfold symmetry is locally broken by a step-induced uniaxial symmetry, $K_{\mathrm{s}}[20,21]$. Weber et al. grew epitaxial (001)Co films on a stepped surface $[20,21]$. While the hysteresis loops were measured along one of the original easy axes, a constant bias field was applied along the other easy axis, perpendicular to the measuring direction. The measured loops then also exhibit a double shifted loop. Step-induced uniaxial anisotropy breaks the biaxial symmetry of Co, and leads to the observed double shifted magnetization curves. The shift field is increased with an increasing bias field. There is an analogy between the exchange bias system of $\mathrm{NiMn} / \mathrm{Co}$ and the system of Co grown on a stepped surface. Exchange coupling between NiMn and Co induces an internal bias field (exchange field) as well as a biquadratic coupling. This biquadratic exchange coupling produces a uniaxial anisotropy along the applied field direction during annealing, and results in double shifted loops along the hard axis.

\section{Asymmetric loops in bilayers}

Some recent studies of magnetic bilayer indicate that magnetization reversal is intrinsically asymmetric under appropriate conditions [69-71]. Various mechanisms have been proposed to explain the formation of asymmetric loops. Asymmetric domain wall motion was believed to be the origin of asymmetric hysteresis loops. However, asymmetric hysteresis loops can also be easily induced with an undirectional bias field as shown by the coherent rotation model. Since the center of the astroid deviates from the center of the external field the nucleation conditions are very asymmetric in the opposite direction of the applied field. Fig. 13 shows asymmetrically shaped hysteresis loops under the biased field along the easy axis. Our calculated results suggest that asymmetric loops observed in various exchange biased bilayers can be explained within the coherent rotation model with a biased astroid center.

\section{Conclusions}

Two different origins of the formation of double shifted magnetization curves in magnetic bilayers have been discussed here. Besides the intrinsic biaxial anisotropy 
Table 2

Shift field for substrate/film combinations resulting in the double shifted magnetization curves (ML: monolayer)

\begin{tabular}{|c|c|c|c|}
\hline Substrate & Film (thickness) & $\begin{array}{l}\text { Shift field (Oe); } \\
\text { Sweep field } \\
\text { direction }\end{array}$ & Ref. \\
\hline $\mathrm{Cu}(001)$ & $\begin{array}{l}\text { Co }(5 \mathrm{ML}) \\
\text { Deposition angle } 20-80^{\circ}\end{array}$ & $\begin{array}{l}45-270 \text {; } \\
\text { deposition direction }\end{array}$ & [15] \\
\hline Stepped Cu, ( $\left(\begin{array}{lll}1 & 1 & 17\end{array}\right)$ & $\begin{array}{l}\text { Co }(4 \mathrm{ML}) \\
\text { Step edges } \|[1-10]\end{array}$ & $\begin{array}{l}57 \\
{[110]}\end{array}$ & [47] \\
\hline Stepped Cu(1 00$)$ & Co $(\sim 4.1 \mathrm{ML})$ & $321(\max )$ & {$[50]$} \\
\hline Miscut angle $0.7^{\circ}$ & Step edges $\|[1-10]$ & {$[110]$} & \\
\hline Stepped $\mathrm{Cu}(001)$ & Co $(8 \mathrm{ML})$ & $0-262$ & [49] \\
\hline Miscut angle $0-5.4^{\circ}$ & Step edges $\|[1-10]$ & [110] & \\
\hline Stepped Mo(001) & $\mathrm{NiFe}(50 \mathrm{~nm})$ & 6 & [55] \\
\hline Miscut angle $3^{\circ}$ & Step edges $\|[1-10]$ & [110] & \\
\hline Stepped $\operatorname{Ag}(001)$ & $\mathrm{Fe}(25 \mathrm{ML})$ & $0-160$ & [16] \\
\hline Miscut angle $0-10^{\circ}$ & Step edges $\|[100]$ & {$[010]$} & \\
\hline Stepped W(001) & $\mathrm{Fe}(2 \mathrm{ML})$ & $0-356$ & \\
\hline Miscut angle $0-8.5^{\circ}$ & Step edges $\|[100]$ & {$[100]$} & [52] \\
\hline \multirow[t]{2}{*}{ GaAS(-101) } & $\mathrm{Fe}(6.6 \mathrm{~nm})$ & $55 ;[001]$ & [53] \\
\hline & $\mathrm{Fe}(14.5,23 \mathrm{~nm})$ & 24,$58 ;[110]$ & \\
\hline $\operatorname{GaAs}(100)$ & bcc $\mathrm{Fe}_{x} \mathrm{Co}_{1-x}(100)$ & 127,177 & [56] \\
\hline Miscut angle $0^{\circ}, 4^{\circ}$ & Step edges $\|[01-1]$ & {$[01-1]$} & \\
\hline ScErAs/GaAs(100) & bcc $\mathrm{Fe}_{x} \mathrm{Co}_{1-x}(100)$ & 50,81 & [56] \\
\hline Miscut angle $4^{\circ}, 10^{\circ}$ & Step edges $\|[01-1]$ & {$[01-1]$} & \\
\hline $\mathrm{ZnSe}(001) / \mathrm{GaAs}(001)$ & FeCo $(10 \mathrm{~nm})$ & $7 ;[1-10]$ & [17] \\
\hline
\end{tabular}

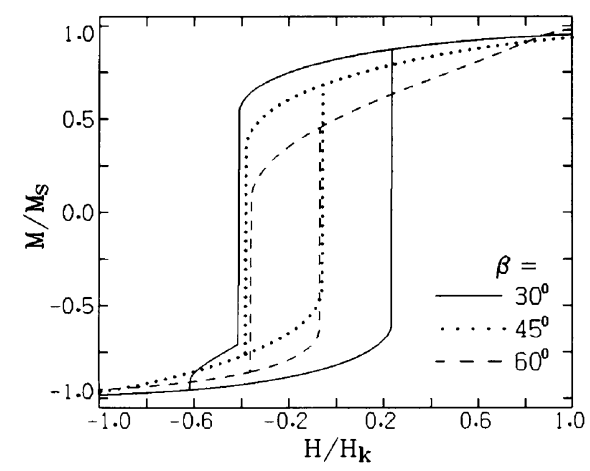

Fig. 13. Calculated hysteresis loops of exchange biased bilayers exhibiting an asymmetric shape. Here $K_{1}>0, \alpha=-1.6$, and $H_{\mathrm{b}} /$ $H_{\mathrm{k}}=0.2$.

within epitaxial magnetic ultrathin films there exists also an induced interfacial uniaxial anisotropy which causes the effective quadratic term to vary in ultrathin film. Magnetic material constants can be tailored by the film thickness, and different type of magnetization curves can then be observed experimentally. The single domain theory of coherent rotation gives a reasonable fit of experimental data on the double shifted magnetization curves. A starlike astroid indicates four metastable states which appear at a large angle away from the hard axis, and the double shifted magnetization curves can thus easily observed in magnetic bilayers.

\section{Acknowledgements}

This work was supported by National Science Council, Taiwan, under Grant No. 89-2119-M002-014 and 89-2112M-019-008.

\section{References}

[1] U. Gradmann, in: K.H.J. Buschow (Ed.), Handbook of Magnetic Materials, North-Holland, New York, 1993.

[2] J.A.C. Bland, B. Heinrich (Eds.), Ultrathin Magnetic Structures I, Springer-Verlag, Berlin, 1994.

[3] P. Bruno, J.P. Renard, Appl. Phys. A49 (1989) 499-506.

[4] R.H. Victora, J.M. MacLaren, Phys. Rev. B47 (1993) 1158311586.

[5] L. Gonzales, R. Miranda, M. Salmeron, J.A. Verges, Yndurain, Phys. Rev. B24 (1981) 3245.

[6] C.R. Chang, Phys. Rev. B48 (1993) 15817-15822.

[7] A. Yamaguchi, S. Ogu, W.H. Soe, R. Yamamoto, Appl. Phys. Lett. 62 (1993) 1020-1022.

[8] M.N. Baibich, J.M. Broto, A. Fert, F. Nguyen Van Dau, F. Petroff, P. Etienne, G. Greuzet, A. Friederich, J. Chazelas, Phys. Rev. Lett. 61 (1988) 2472-2475.

[9] G.A. Prinz, Phys. Today 48 (1995) 58.

[10] J. Nogués, I.K. Schuller, J. Magn. Magn. Mater. 192 (1999) 203-232.

[11] A.E. Berkowitz, K. Takano, J. Magn. Magn. Mater. 200 (1999) 552-570.

[12] C.-R. Chang, D.R. Fredkin, J. Appl. Phys. 63 (1988) 34353437. 
[13] C.-R. Chang, J. Appl. Phys. 69 (1991) 2431-2439.

[14] W. Weber, C.H. Back, A. Bischof, D. Pescia, R. Allenspach, Nature 374 (1995) 788-790.

[15] S. van Dijken, G.D. Santo, B. Poelsema, Appl. Phys. Lett. 77 (2000) 2030-2032.

[16] R.K. Kawakami, E.J. Escorcia-Apariacio, Z.Q. Qiu, Phys. Rev. Lett. 77 (1996) 2570-2573.

[17] C.J. Gutierrez, J.J. Krebs, G.A. Prinz, Appl. Phys. Lett. 61 (1992) 2476-2478.

[18] M. Albrecht, T. Furubayashi, M. Przybylski, J. Korecki, U. Gradmann, J. Magn. Magn. Mater. 113 (1992) 207-220.

[19] C.K. Lo, Y. Liou, C.P. Chang, I. Klik, Y.D. Yao, J.C.A. Huang, Appl. Phys. Lett. 68 (1996) 2155-2157.

[20] W. Weber, C.H. Back, A. Bischof, Ch. Würsch, R. Allenspach, Phys. Rev. Lett. 76 (1996) 1940-1943.

[21] W. Weber, R. Allenspach, A. Bischof, Appl. Phys. Lett. 70 (1997) 520-522.

[22] T. Leeb, M. Brockmann, F. Bensch, S. Miethaner, G. Bayreuther, J. Appl. Phys. 85 (1999) 4964-4966.

[23] R.P. Michel, A. Chaiken, C.T. Wang, L.E. Johnson, Phys. Rev. B58 (1998) 8566-8573.

[24] Y.J. Tang, X. Zhou, X. Chen, B.Q. Liang, W.S. Zhan, J. Appl. Phys. 88 (2000) 2054-2057.

[25] W.F. Brown Jr, Micromagnetics, Krieger, New York, 1978.

[26] A. Aharoni, Introduction to the Theory of Ferromagnetism, Clarendon, Oxford, 1996.

[27] L. Landau, E. Lifshitz, Physik A, Soviet Union 8 (1935) 153.

[28] W.F. Brown Jr, J. Phys. Rev. 58 (1940) 736-743.

[29] W.F. Brown Jr, J. Phys. Rev. 105 (1957) 1479.

[30] W.F. Brown Jr, J. Appl. Phys. 49 (1978) 1937-1942.

[31] W.F. Brown Jr, Rev. Mod. Phys. 17 (1945) 15-19.

[32] J. Fidler, S. Schrefl, J. Phys. D: Appl. Phys. 33 (1999) R135R156.

[33] E.C. Stoner, E.P. Wohlfarth, Philos. Trans. Roy. Soc. London A240 (1948) 599-642.

[34] E.C. Stoner, E.P. Wohlfarth, IEEE Trans. Magn. 27 (1991) 3475-3518 (reprint).

[35] G. Herzer, W. Fernengel, E. Adler, J. Magn. Magn. Mater. 58 (1986) 48-54.

[36] H. Kronmüller, K.-D. Durst, G. Martinek, J. Magn. Magn. Mater. 69 (1987) 149-157.

[37] J.C. Oliverira de Jesus, W. Kleemann, J. Magn. Magn. Mater. 169 (1997) 159-168.

[38] Y.T. Millev, J.R. Cullen, H.P. Oepen, J. Appl. Phys. 83 (1998) 6500-6502.

[39] A. Aharoni, S. Shtrikman, Phys. Rev. 109 (1958) 1522-1528.

[40] A. Aharoni, J. Magn. Magn. Mater. 196-197 (1999) 786-790.

[41] U. Hartmann, Phys. Rev. B36 (1987) 2331-2332.

[42] H. Kronmüller, K.-D. Durst, M. Sagawa, J. Magn. Magn. Mater. 74 (1988) 291-302.

[43] L. Vatsitchev, Phys. Stat. Sol. (a) 67 (1981) 591-596.

[44] M. Labrune, J.C.S. Kools, A. Thiaville, J. Magn. Magn. Mater. 171 (1997) 1-15.
[45] J.P. King, J.N. Champman, J.C.S. Kools, J. Magn. Magn. Mater. 177-181 (1998) 896-897.

[46] J.P. King, J.N. Champman, J.C.S. Kools, M.F. Gillies, J. Phys. D: Appl. Phys. 32 (1999) 1087-1096.

[47] W. Wulfhekel, S. Knappmann, H.P. Oepen, J. Magn. Magn. Mater. 163 (1996) 267-276.

[48] W. Weber, A. Bischof, R. Allenspach, Ch. Würsch, C.H. Back, D. Pescia, Phys. Rev. Lett. 76 (1996) 3424-3427.

[49] R.K. Kawakami, M.O. Bowen, H.J. Choi, E.J. Escorcia-Aparicio, Z.Q. Qiu, Phys. Rev. B58 (1998) R5924-R5927.

[50] A. Rettori, L. Trallori, M.G. Pini, C. Stamm, Ch. Würsch, S. Egger, D. Pescia, IEEE Trans. Magn. 34 (1998) 1195-1199.

[51] Y.H. Wang, C.H. Lai, C.-R. Chang, J.-S. Yang, submitted to Phys. Rev. B.

[52] H.J. Choi, Z.Q. Qiu, J. Pearson, J.S. Jiang, D. Li, S.D. Bader, Phys. Rev. B57 (1998) R12713-R12716.

[53] M. Gester, C. Daboo, R.J. Hicken, S.J. Gray, J.A.C. Bland, Thin Solid Film 275 (1996) 91-94.

[54] E.J. Escorcia-Apariacio, H.J. Choi, W.L. Ling, R.K. Kawakami, Z.Q. Qiu, Phys. Rev. Lett. 81 (1998) 2144-2147.

[55] C.C. Yu, PhD Thesis, National Cheng-Kung University, Taiwan, 2000.

[56] L.C. Chen, J.W. Dong, B.D. Schultz, C.J. Palmstron, J. Berezovsky, A. Isakovic, P.A. Crowell, N. Tabat, J. Vac. Sci. Technol. B18 (2000) 2057-2062.

[57] L. Néel, J. Phys. Rad. 15 (1954) 225.

[58] L. Néel, Compt. Rend. 237 (1953) 1468.

[59] S. Chikazumi, Physics of Magnetism, New York (1964) 164.

[60] W. Keune, R. Holbauer, U. Gonser, J. Lauer, D.L. Williamson, J. Appl. Phys. 48 (1977) 2976.

[61] G.A. Prinz, Phys. Rev. Lett. 54 (1985) 1051-1054.

[62] J.C.A. Huang, Y.M. Hu, C.C. Yu, C.H. Tsao, C.H. Lee, Phys. Rev. B57 (1998) 11517-11520.

[63] W.A. Jesser, D. Kuhlmann-Wilsdorf, Phys. Stat. Sol. 19 (1967) 65.

[64] D.S. Chuang, C.A. Ballentine, R.C. O’Handley, Phys. Rev. B49 (1994) 15084-15095.

[65] R.R. Du, C.P. Flynn, J. Phys. Condens. Matter 2 (1990) 1338.

[66] J.C.A. Huang, R.R. Du, C.P. Flynn, Phys. Rev. B44 (1991) 4060.

[67] C.H. Lai, S.A. Chen, J.C.A. Huang, J. Magn, Magn. Mater. 209 (2000) 122-124.

[68] Y.H. Wang, C.H. Lai, C.K. Lo, Y.D. Yao, J. Magn. Magn. Mater. 209 (2000) 119-121.

[69] V.I. Nikitenko, V.S. Gornakov, A.J. Shapiro, R.D. Shull, K. Liu, S.M. Zhou, C.L. Chien, Phys. Rev. Lett. 84 (2000) 765768.

[70] M.R. Fitzsimmons, P.C. Yashar, C. Leighton, J. Nogués, J. Dura, C.F. Majkrzak, I.K. Schuller, Phys. Rev. Lett. 84 (2000) 3986-3999.

[71] C. Leighton, M. Song, J. Nogués, M.C. Cyrille, I.K. Schuller, J. Appl. Phys. 88 (2000) 344-347.

[72] G.A. Prinz, Science 282 (1998) 1660-1663. 